\title{
Autorenkollektiv
}

Wissenschaftliche Schulen Band 2 


\section{WISSENSCHAFT \\ UND \\ GESELLSCHAFT}

\author{
Herausgegeben \\ vom Institut \\ für Theorie, Geschichte \\ und Organisation \\ der Wissenschaft der \\ Akademie der Wissenschaften \\ der DDR \\ durch \\ Günter Kröber
}

Band 11-2 
Akademie der Wissenschaften der UdSSR Institut für Geschichte der Naturwissenschaft und Technik

Akademie der Wissenschaften der DDR Institut für Theorie, Geschichte und Organisation der Wissenschaft

\title{
Wissenschaftliche Schulen
}

\section{Band 2}

\author{
Herausgegeben von \\ Semen R. Mikulinskij, \\ Michail G.Jaroševskij, \\ Helmut Steiner und \\ Rose-Luise Winkler \\ unter Mitarbeit von Peter Altner
}

AKADEMIE - VERLAG - BERLIN

1979 
Titel der sowjetischen Ausgabe:

школы в вауке

Москва 1977 - Издательство „Наука“

Herausgegeben von:

S. R. Mikulinskij, M. G. Jaroševskij,

G. Kröber, H. Steiner

Erschienen in der Reihe:

Науковедение: Проблемы и исследования

Redaktionskollegium der Reihe:
D. M. Gvišiani
A. M. Rumjancev
N. M. Zavoronkov
V. N. Stoletov
B. M. Kedrov
V. A. Filippov
V. P. Kuz'min
M. G. Jaroševskij
S. R. Mikulinskij (Leiter)
A. V. Pilipenko (verantwortlicher Redakteur)

Ersohienen im Akademie -Verlag, DDR - 108 Berlin, Leipziger Str. 3-4

Lektor: Dr. Dorothea Fensch

(c) Akademie-Verlag Berlin 1979

Lizenznummer: $202 \cdot 100 / 50 / 79$

Umschlaggestaltung: Rolf Kunze

Gesamtherstellung: IV/2/14 VEB Druckerei \&Gottfried Wilhelm Leibniz,,

445 Gräfenhainichen · 5314

Bestellnummer: $7536064(2162 / 11 / 2) \cdot \mathrm{LSV} 0305$

Printed in GDR

DDR 12,50 